Research Article

\title{
Estimating the PI-Estrada index of graphs
}

\author{
Akbar Jahanbani ${ }^{1}$, Hajar Shooshtari ${ }^{1}$, Jonnathan Rodríguez ${ }^{2, *}$ \\ ${ }^{1}$ Department of Mathematics, Azarbaijan Shahid Madani University, Tabriz, Iran \\ ${ }^{2}$ Departamento de Matemáticas, Facultad Ciencias Básicas, Universidad de Antofagasta, Av. Angamos 0601 Antofagasta, Chile
}

(Received: 7 November 2020. Received in revised form: 12 February 2021. Accepted: 1 March 2021. Published online: 5 March 2021.)

(c) 2021 the authors. This is an open access article under the CC BY (International 4.0) license (www.creativecommons.org/licenses/by/4.0/)

\begin{abstract}
Let $G$ be a graph with $n$ vertices. The PI-Estrada index of $G$ is an invariant that is calculated from the eigenvalues of the vertex-PI matrix of $G$. The main purpose of this paper is to establish upper and lower bounds for the PI-Estrada index of a graph in terms of the number of vertices, edges, triangles and pendant vertices.
\end{abstract}

Keywords: PI Matrix; PI-Estrada index; PI-energy.

2020 Mathematics Subject Classification: 05C50.

\section{Introduction}

Let $G=(V, E)$ be a simple undirected graph with vertex set $V(G)$ and edge set $E(G)$. The integers $n=|V|$ and $m=|E|$ are the number of vertices and edges of the graph $G$, respectively. Let $U$ be a subset of $V(G)$. We denote by $\langle U\rangle$ the subgraph of $G$ induced by $U$. A clique of $G$ is a subset of mutually adjacent vertices of $V(G)$. A clique is called maximal if it is not contained in any other clique. A clique is said to be maximum if it has the maximum cardinality. The size of a maximum clique in $G$ is called the clique number of $G$ and is denoted by $\omega(G)$. A walk from a vertex $u$ to a vertex $v$ is a finite alternating sequence $v_{0}(=u) e_{1} v_{1} e_{2} \ldots v_{k-1} e_{k} v_{k}(=v)$ of vertices and edges such that $e_{i}=v_{i-1} v_{i}$ for $i=1,2, \ldots, k$ where the number $k$ is the length of the walk. A graph is connected if each pair of vertices in a graph is joined by at least one walk. As usual, denote by $P_{n}, K_{n}$ and $\bar{K}_{n}$ the path, the complete graph, and the complement of complete graph with $n$ vertices, respectively. The distance between two arbitrary vertices $u$ and $v$ of $G$, denoted by $d(u, v)$, is defined as the number of edges in the shortest path connecting the vertices $u$ and $v$. The adjacency matrix $A(G)$ of $G$ is a matrix with entries $a_{i j}=1$ if $v_{i} v_{j} \in E(G)$ and 0 otherwise. We denote by $\lambda_{1}, \lambda_{2}, \ldots, \lambda_{n}$ the eigenvalues of $A(G)$.

The Estrada index, put forward by Ernesto Estrada [11], is defined as

$$
E E=E E(G)=\sum_{i=1}^{n} e^{\lambda_{i}}
$$

The Estrada index is used to quantify the degree of folding of long-chain molecules, especially proteins [11] and complex networks [29-31]. Additional applications of the Estrada index can be found in [7,12-15].

The mathematical aspects of the Estrada index have been intensively studied. Several upper and lower bounds and new Estrada indices were obtained in [1,2,25-28,32]. One of the most important properties of the Estrada index is as following:

$$
E E=\sum_{k=1}^{\infty} \frac{M_{k}(G)}{k !}
$$

where $M_{k}=M_{k}(G)$ is the $k$-th moment of the graph $G$, i.e.,

$$
M_{k}=M_{k}(G)=\sum_{i=1}^{n} \lambda_{i}^{k}
$$

It is well known that $M_{k}(G)$ is equal to the number of closed walks of length $k$ in $G$.

The investigation of topological indices has been shown to give a high degree of predictability of pharmaceutical properties. Among the several existing graph-based molecular structure descriptors [33,34], the Randić index is certainly the most widely applied in chemistry and pharmacology, in particular for designing in the quantitative structure-activity 
relationship models (QSPR) used in the chemical, biological science, and quantitative structure-property relationships (QSAR). Due to the importance and practicality of these topological indices, Bozkurt et al. [6] in 2012, introduced the Randić energy and the Randić Estrada index of a graph, which has been of interest to many researchers.

Let $e=u v$ be an edge of $G$. Then, $n_{u}(e \mid G)$ is the number of edges lying closer to $u$ than to $v$ and $n_{v}(e \mid G)$ is defined analogously. The Padmakar-Ivan (PI) index of a graph $G$ is defined [21] as

$$
\mathbf{P I}(G)=\sum_{u v \in E(G)}\left(n_{u}(e \mid G)+n_{v}(e \mid G)\right) .
$$

For details on this invariant, see some of the most cited papers [19-22,24]. The PI index promises to be a useful parameter in the QSPR/QSAR studies. A more favorable comparison with other representative indices such as the Randić index has already been made in order to establish the predictive ability of the PI index, and the results have shown that in several cases the PI index gave better results. Considering the importance of this index and being better than the Randić index, Arani [3] in 2011, introduced the PI-energy. In this paper, we introduce the PI-Estrada index and examine some of its properties.

The vertex-PI matrix (see [3]) of $G$, denoted by $P I(G)$, is defined as a matrix whose $(i, j)$-entry is $d_{i j}$, where

$$
d_{i j}= \begin{cases}n_{u_{i}}(e \mid G)+n_{v_{j}}(e \mid G) & \text { if } u_{i} v_{j} \in E(G) \\ 0 & \text { otherwise }\end{cases}
$$

Since $P I(G)$ is a real and symmetric matrix, its eigenvalues are real numbers, and we label them in non-increasing order by $\delta_{1} \geqslant \delta_{2} \geqslant \ldots \geqslant \delta_{n}$. Their collection is called the PI-spectrum of $G$. The spectral radius of $G$, denoted by $\delta_{1}(G)$, is the largest eigenvalue of $P I(G)$.

A spectral invariant of the matrix $P I(G)$ is the vertex-PI energy of $G$, which is defined [3] as

$$
E_{P I}=E_{P I}(G)=\sum_{i=1}^{n}\left|\delta_{i}\right| .
$$

Recently, several analogous concepts such as Zagreb Estrada index [26], Harmonic Estrada index [18], Albertson-Estrada index [16] and Hermitian Estrada index [17] of graphs and digraphs were put forward.

On the other hand, the vertex-PI index is a distance-based molecular structure descriptor, that recently found numerous chemical applications. In this paper, we introduce the PI-Estrada index and denote it by $E E_{P I}$.

Definition 1.1. Let $G$ be a graph of order $n$ whose PI-eigenvalues are $\delta_{1} \geqslant \delta_{2} \geqslant \ldots \geqslant \delta_{n}$. The PI-Estrada index of $G$ is defined as

$$
E E_{P I}=E E_{P I}(G)=\sum_{i=1}^{n} e^{\delta_{i}}
$$

Also, we define

$$
N_{k}=\sum_{i=1}^{n} \delta_{i}^{k}
$$

Similar to the Estrada index in (1), we have

$$
E E_{P I}(G)=\sum_{k=1}^{\infty} \frac{N_{k}}{k !}
$$

This paper is organized as follows. In Section 2 , we give a list of some previously known results. In Section 3, we establish upper and lower bounds for the PI-Estrada index.

\section{Preliminaries and known results}

In this section, we give some preliminary results useful for the proof of the our results. Arani et al. [4], proved the following result.

Lemma 2.1. [4] Let $G$ be a graph with n vertices. Then

$$
\delta_{1} \geqslant \frac{2 \boldsymbol{P I}}{n}
$$

Deng [9], showed the following result. 
Lemma 2.2. [9] Let $G$ be a simple connected graph with $m$ edges. Then

$$
\boldsymbol{P I}(G) \geqslant \boldsymbol{M}_{1}(G)-2 m
$$

where $\boldsymbol{M}_{1}(G)=\sum_{i=1}^{n} d_{i}^{2}$.

In [8], Das et al. obtained the following result.

Lemma 2.3. [8] Let $G$ be a connected graph with $n$ vertices, $m$ edges, $p$ pendent vertices, and clique number $\omega(\omega \geqslant 3)$. Then

$$
\boldsymbol{P I}(G) \geqslant 2 m+(n-2) p+(n-\omega)(\omega-1) .
$$

Khadikar et al. [23], proved the following results.

Lemma 2.4. [23] Let $T$ be a tree with $n$ vertices, $n \geqslant 2$. Then

$$
\boldsymbol{P I}(T)=(n-1)(n-2) .
$$

Lemma 2.5. [23] Let $C_{n}$ be a cycle graph with $n \geqslant 3$. Then

$$
\begin{gathered}
\boldsymbol{P I}\left(C_{2 n+1}\right)=2 n(2 n+1), \\
\boldsymbol{P I}\left(C_{2 n}\right)=4 n(n-1) .
\end{gathered}
$$

Lemma 2.6. [5] For any real $x$, one has $e^{x} \geqslant 1+x+\frac{x^{2}}{2}+\frac{x^{3}}{3 !}$. Equality holds if and only if $x=0$.

Lemma 2.7. For any non-negative numbers $x_{1}, x_{2}, \ldots, x_{n}$ and $k \geqslant 2$, we have

$$
\sum_{i=1}^{n}\left(x_{i}\right)^{k} \leqslant\left(\sum_{i=1}^{n} x_{i}^{2}\right)^{\frac{k}{2}}
$$

Remark 2.1. For any real $x$, the power-series expansion of $e^{x}$, is the following

$$
e^{x}=\sum_{k \geqslant 0} \frac{x^{k}}{k !}
$$

The following results come from [4].

Lemma 2.8. [4] Let $G$ be a graph with $n$ vertices, $m$ edges, $t$ triangles and $\delta_{1}, \delta_{2}, \ldots, \delta_{n}$ be a non-increasing arrangement of PI-eigenvalues of $G$. Then

$$
\begin{aligned}
& \text { 1) } \sum_{i=1}^{n} \delta_{i}=0, \\
& \text { 2) } \quad 8 m \leqslant \sum_{i=1}^{n} \delta_{i}^{2} \leqslant 2 m n^{2}, \\
& \text { 3) } 24 t \leqslant \sum_{i=1}^{n} \delta_{i}^{3} \leqslant 6 t n^{3} .
\end{aligned}
$$

Lemma 2.9. [4] Let $G$ be a bipartite graph with $n$ vertices. Then

$$
E_{P I}(G) \leqslant n \sqrt{2 m n}
$$

Lemma 2.10. [4] Let $G$ be a graph with $n$ vertex and $P I$ degree sequence $\left\{P_{1}, P_{2}, \ldots, P_{n}\right\}$. Then

$$
\delta_{1} \geqslant \sqrt{\frac{P_{1}^{2}+P_{2}^{2}+\ldots+P_{n}^{2}}{n}} .
$$

Lemma 2.11. [4] Let $G$ be a graph with $n$ vertex, $P I$ degree sequence $\left\{P_{1}, P_{2}, \ldots, P_{n}\right\}$ and second vertex PI degree sequence $\left\{T_{1}, T_{2}, \ldots, T_{n}\right\}$. Then

$$
\delta_{1} \geqslant \sqrt{\frac{T_{1}^{2}+T_{2}^{2}+\ldots+T_{n}^{2}}{P_{1}^{2}+P_{2}^{2}+\ldots+P_{n}^{2}}}
$$




\section{Main results}

In this section, we present upper and lower bounds for the PI-Estrada index in terms of the number of vertices, edges, triangles, a bound in terms of the elements of the degree sequence, pendent vertices and clique number for a graph, connected graphs, cycle graphs and tree graphs. Our first main result is the following.

Theorem 3.1. Let $G$ be a graph with $n$ vertices, $m$ edges and $t$ triangles. Then

$$
E E_{P I}(G) \geqslant \sqrt{n^{2}+8 n(t+m)} .
$$

Equality holds if and only if $G$ is the empty graph $\bar{K}_{n}$.

Proof. Suppose that $\delta_{1}, \delta_{2}, \ldots, \delta_{n}$ form the spectrum of the $P I$ matrix. By definition of the $P I$-Estrada index and Lemma 2.6, we have

$$
\begin{aligned}
E E_{P I}(G)^{2} & =\sum_{i=1}^{n} \sum_{j=1}^{n} e^{\delta_{i}+\delta_{j}} \\
& \geqslant \sum_{i=1}^{n} \sum_{j=1}^{n}\left(1+\delta_{i}+\delta_{j}+\frac{\left(\delta_{i}+\delta_{j}\right)^{2}}{2}+\frac{\left(\delta_{i}+\delta_{j}\right)^{3}}{6}\right) \\
& =\sum_{i=1}^{n} \sum_{j=1}^{n}\left(1+\delta_{i}+\delta_{j}+\frac{\delta_{i}^{2}}{2}+\frac{\delta_{j}^{2}}{2}+\delta_{i} \delta_{j}+\frac{\delta_{i}^{3}}{6}+\frac{\delta_{j}^{3}}{6}+\frac{\delta_{i}^{2} \delta_{j}}{2}+\frac{\delta_{i} \delta_{j}^{2}}{2}\right) .
\end{aligned}
$$

Now, by Equality (5),

$$
\begin{gathered}
\sum_{i=1}^{n} \sum_{j=1}^{n}\left(\delta_{i}+\delta_{j}\right)=n \sum_{i=1}^{n} \delta_{i}+n \sum_{j=1}^{n} \delta_{j}=0 . \\
\sum_{i=1}^{n} \sum_{j=1}^{n} \delta_{i} \delta_{j}=\left(\sum_{i=1}^{n} \delta_{i}\right)^{2}=0 .
\end{gathered}
$$

By Inequality (6),

$$
\sum_{i=1}^{n} \sum_{j=1}^{n}\left(\frac{\delta_{i}^{2}}{2}+\frac{\delta_{j}^{2}}{2}\right)=\frac{n}{2} \sum_{i=1}^{n} \delta_{i}^{2}+\frac{n}{2} \sum_{j=1}^{n} \delta_{j}^{2}=n \sum_{i=1}^{n} \delta_{i}^{2} \geqslant 8 m n .
$$

Also, by Equality (5) and Inequality (6), it holds that

$$
\sum_{i=1}^{n} \sum_{j=1}^{n} \frac{\delta_{i}^{2} \delta_{j}}{2}=\sum_{i=1}^{n} \sum_{j=1}^{n} \frac{\delta_{i} \delta_{j}^{2}}{2}=0 .
$$

Similarly by inequality (7),

$$
\sum_{i=1}^{n} \sum_{j=1}^{n}\left(\frac{\delta_{i}^{3}}{6}+\frac{\delta_{j}^{3}}{6}\right)=\frac{n}{6} \sum_{i=1}^{n} \delta_{i}^{3}+\frac{n}{6} \sum_{j=1}^{n} \delta_{j}^{3}=\frac{n \sum_{j=1}^{n} \delta_{j}^{3}}{3} \geqslant 8 n t .
$$

Combining the above relations, we get

$$
E E_{P I}(G) \geqslant \sqrt{n^{2}+8 n(t+m)} .
$$

So, the inequality of the theorem is proved. The equality holds if and only if all $\delta_{i}$ are zero that is $G \cong \bar{K}_{n}$.

Theorem 3.2. Let $G$ be a graph with $n$ vertices, $m$ edges and $t$ triangles. Then

$$
E E_{P I}(G) \geqslant n+8 m+(\sinh (1)-1) 24 t+(\cosh (1)-1) N_{4},
$$

where $N_{4}=\sum_{i=1}^{n} \delta_{i}^{4}$. Equality in (9) holds if and only if $G$ is the empty graph $\bar{K}_{n}$.

Proof. Note that $N_{0}=\sum_{i=1}^{n} \delta_{i}^{0}=n, N_{1}=\sum_{i=1}^{n} \delta_{i}=0$ and $N_{2}=\sum_{i=1}^{n} \delta_{i}^{2} \geqslant 8 m$. Therefore, by definition of the PI-Estrada index, we have

$$
E E_{P I}(G)=\sum_{k \geqslant 0} \frac{N_{k}}{k !} \geqslant n+8 m+\sum_{k \geqslant 1} \frac{N_{2 k+1}}{(2 k+1) !}+\sum_{k \geqslant 1} \frac{N_{2 k+2}}{(2 k+2) !}
$$




$$
\begin{aligned}
& \geqslant n+8 m+\sum_{k \geqslant 1} \frac{N_{3}}{(2 k+1) !}+\sum_{k \geqslant 1} \frac{N_{4}}{(2 k+2) !} \\
& \geqslant n+8 m+(\sinh (1)-1) 24 t+(\cosh (1)-1) N_{4} .
\end{aligned}
$$

The result follows easily. The equality holds if and only if all $\delta_{i}$ are zero that is $G \cong \bar{K}_{n}$.

Remark 3.1. Note that the above result can be written as:

$$
E E_{P I}(G) \geqslant n+8 m+4.2 t+0.54 N_{4}
$$

Theorem 3.3. Let $G$ be a graph with $n$ vertices and medges. Then for any integer $k_{0} \geqslant 2$,

$$
E E_{P I}(G) \leqslant n-1-n \sqrt{2 m}+\sum_{k=2}^{k_{0}} \frac{N_{k}(G)-(n \sqrt{2 m})^{k}}{k !}+e^{n \sqrt{2 m}} .
$$

Equality holds if and only if $G \cong \bar{K}_{n}$.

Proof. Note that $\sum_{i=1}^{n} \delta_{i}^{2} \leqslant 2 m n^{2}$. By definition of the PI-Estrada index, we have

$$
\begin{aligned}
E E_{P I}(G) & =\sum_{k=0}^{k_{0}} \frac{N_{k}(G)}{k !}+\sum_{k \geqslant k_{0}+1} \frac{1}{k !} \sum_{i=1}^{n} \delta_{i}^{k} \\
& \leqslant \sum_{k=0}^{k_{0}} \frac{N_{k}(G)}{k !}+\sum_{k \geqslant k_{0}+1} \frac{1}{k !} \sum_{i=1}^{n}\left|\delta_{i}^{k}\right| \\
& \leqslant \sum_{k=0}^{k_{0}} \frac{N_{k}(G)}{k !}+\sum_{k \geqslant k_{0}+1} \frac{1}{k !}\left(\sum_{i=1}^{n} \delta_{i}^{2}\right)^{\frac{k}{2}}, \text { by Inequality (3) } \\
& =\sum_{k=0}^{k_{0}} \frac{N_{k}(G)}{k !}+\sum_{k \geqslant k_{0}+1} \frac{(n \sqrt{2 m})^{k}}{k !} \\
& =\sum_{k=0}^{k_{0}} \frac{N_{k}(G)}{k !}+e^{n \sqrt{2 m}}-\sum_{k=0}^{k_{0}} \frac{(n \sqrt{2 m})^{k}}{k !}
\end{aligned}
$$

Considering $k_{0}=2$ in (12), we have the following result that only depends on number of vertices and edges of the graph. If $G \cong \bar{K}_{n}$ it is easy to check that the equality in (10) holds. Suppose now that the equality holds in (10). Then all the inequalities in the proof must be equalities. From Equation (3), we know that is valid if $k$ even or all eigenvalues are negative, but we know that $k$ cannot be just even, so all eigenvalues must be negative, by equality (5), we have $\sum_{i=1}^{n} \delta_{i}=0$. Therefore, we get $\delta_{1}=\delta_{2}=\ldots=\delta_{n}=0$, hence $G \cong \bar{K}_{n}$.

Now, by using Theorem 3.3, we get the next result.

Corollary 3.1. Let $G$ be a graph with $n$ vertices and $m$ edges. Then

$$
E E_{P I}(G) \leqslant n-1-n \sqrt{2 m}+e^{n \sqrt{2 m}} .
$$

Lemma 3.1. Let $G$ be a graph with $n$ vertices and $m$ edges. Then

$$
\delta_{1} \geqslant \frac{8 m}{n \sqrt{2 n m}} .
$$

Equality holds if and only if $G \cong K_{2}$.

Proof. Let $a_{i}, b_{i}$ are decreasing non-negative sequences with $a_{1}, b_{1} \neq 0$ and $w_{i}$ a nonnegative sequence, for $i=1,2, \ldots, n$. The following inequality is valid (see [10], p. 85)

$$
\sum_{i=1}^{n} w_{i} a_{i}^{2} \sum_{i=1}^{n} w_{i} b_{i}^{2} \leqslant \max \left\{b_{1} \sum_{i=1}^{n} w_{i} a_{i}, a_{1} \sum_{i=1}^{n} w_{i} b_{i}\right\} \sum_{i=1}^{n} w_{i} a_{i} b_{i}
$$


For $a_{i}=b_{i}:=\left|\delta_{i}\right|$, and $w_{i}:=1, i=1,2, \ldots, n$, Inequality (14) becomes

$$
\sum_{i=1}^{n} \delta_{i}^{2} \sum_{i=1}^{n} \delta_{i}^{2} \leqslant \max \left\{\delta_{1} \sum_{i=1}^{n}\left|\delta_{i}\right|, \delta_{1} \sum_{i=1}^{n}\left|\delta_{i}\right|\right\} \sum_{i=1}^{n} \delta_{i}^{2}
$$

Since

$$
\sum_{i=1}^{n}\left|\delta_{i}\right|=E_{P I}(G)
$$

Then

$$
\delta_{1} \geqslant \frac{\sum_{i=1}^{n} \delta_{i}^{2}}{E_{P I}(G)}
$$

Therefore, by Lemma 2.9 and Inequality (6), we have

$$
\delta_{1} \geqslant \frac{8 m}{n \sqrt{2 n m}}
$$

It is not difficult to see that equality holds in (13) if $G \cong K_{2}$.

Theorem 3.4. Let $G$ be a graph with $n$ vertices and $m$ edges. Then

$$
E E_{P I}(G) \geqslant e^{\frac{8 m}{n \sqrt{2 n m}}}+\frac{n-1}{e^{\frac{8 m}{n-1}}} .
$$

Equality holds if and only if $G \cong K_{2}$.

Proof. By definition of the PI-Estrada index and using arithmetic-geometric mean inequality, we obtain

$$
\begin{aligned}
E E_{P I}(G) & =e^{\delta_{1}}+e^{\delta_{2}}+\cdots+e^{\delta_{n}} \\
& \geqslant e^{\delta_{1}}+(n-1)\left(\prod_{i=2}^{n} e^{\delta_{i}}\right)^{\frac{1}{n-1}} \\
& =e^{\delta_{1}}+(n-1) e^{\frac{\sum_{i=2}^{n} \delta_{i}}{n-1}} \\
& =e^{\delta_{1}}+(n-1) e^{\frac{-\delta_{1}}{n-1}}
\end{aligned}
$$

Now, consider the following function

$$
f(x)=e^{x}+\frac{n-1}{e^{\frac{x}{n-1}}} .
$$

It is straightforward verified that $f$ is an increasing function for $x>0$. From the Lemma 3.1, we obtain

$$
E E_{P I}(G) \geqslant e^{\frac{8 m}{n \sqrt{2 n m}}}+\frac{n-1}{e^{\frac{8 m}{n-1}}} .
$$

It is not difficult to see that equality holds in (15) if $G \cong K_{2}$.

Analogous, Theorem 3.4 also by Lemmas 2.10 and 2.11, we have the next results.

Lemma 3.2. Let $G$ be a graph with $n$ vertices. Then

$$
E E_{P I}(G) \geqslant e^{\sqrt{\frac{P_{1}^{2}+P_{2}^{2}+\ldots+P_{n}^{2}}{n}}}+\frac{n-1}{e^{\frac{\sqrt{\frac{P_{1}^{2}+P_{2}^{2}+\ldots+P_{n}^{2}}{n}}}{n-1}}}
$$

Lemma 3.3. Let $G$ be a graph with $n$ vertices. Then

$$
E E_{P I}(G) \geqslant e^{\sqrt{\frac{T_{1}^{2}+T_{2}^{2}+\ldots+T_{n}^{2}}{P_{1}^{2}+P_{2}^{2}+\ldots+P_{n}^{2}}}}+\frac{n-1}{e^{\frac{\sqrt{\frac{T_{1}^{2}+T_{2}^{2}+\ldots+T_{n}^{2}}{P_{1}^{2}+P_{2}^{2}+\ldots+P_{n}^{2}}}}{n-1}}}
$$


Now, by combining Lemma 2.1 with Lemmas $2.2,2.3,2.4$, and 2.5, we give lower bounds for the PI-Estrada index of connected graphs, cycle graphs and tree graphs.

Corollary 3.2. Let $G$ be a connected graph with $n$ vertices, $m$ edges, $p$ pendent vertices, and clique number $\omega(\omega \geqslant 3)$. Then

$$
\begin{aligned}
& \text { 1) } E E_{P I}(G) \geqslant e^{\boldsymbol{M}_{1}(G)-2 m}+\frac{n-1}{e^{\frac{\boldsymbol{M}_{1}(G)-2 m}{n-1}}} \\
& \text { 2) } E E_{P I}(G) \geqslant e^{2 m+(n-2) p+(n-\omega)(\omega-1)}+\frac{n-1}{e^{\frac{2 m+(n-2) p+(n-\omega)(\omega-1)}{n-1}}} .
\end{aligned}
$$

Corollary 3.3. Let $C_{n}$ be a cycle graph with $n \geqslant 3$ vertices. Then

$$
\begin{aligned}
& \text { 1) } E E_{P I}\left(C_{2 n+1}\right) \geqslant e^{4(n-1)}+\frac{n-1}{e^{4}} \\
& \text { 2) } E E_{P I}\left(C_{2 n}\right) \geqslant e^{2(2 n+1)}+\frac{n-1}{e^{\frac{2(2 n+1)}{n-1}}} .
\end{aligned}
$$

Corollary 3.4. Let $T$ be a tree with $n$ vertices, $n \geqslant 2$. Then

$$
E E_{P I}\left(T_{n}\right) \geqslant e^{(n-1)(n-2)}+\frac{n-1}{e^{(n-2)}} .
$$

Theorem 3.5. Let $G$ be a non-empty graph with $n$ vertices and $m$ edges. Then

$$
E E_{P I}(G)<e^{n \sqrt{2 m}} .
$$

Proof. By definition of the PI-Estrada index and Inequalities (3) and (6), we have

$$
\begin{aligned}
E E_{P I}(G)=\sum_{i=1}^{n} e^{\delta_{i}}<\sum_{i=1}^{n} e^{\left|\delta_{i}\right|} & =\sum_{i=1}^{n} \sum_{k \geqslant 0} \frac{\left(\left|\delta_{i}\right|\right)^{k}}{k !} \\
& =\sum_{k \geqslant 0} \frac{1}{k !} \sum_{i=1}^{n}\left(\left|\delta_{i}\right|\right)^{k} \\
& \leq \sum_{k \geqslant 0} \frac{1}{k !}\left(\sum_{i=1}^{n}\left(\left|\delta_{i}\right|\right)^{2}\right)^{\frac{k}{2}} \\
& =\sum_{k \geqslant 0} \frac{1}{k !}\left(\sum_{i=1}^{n}\left(\delta_{i}\right)^{2}\right)^{\frac{k}{2}} \\
& =\sum_{k \geqslant 0} \frac{1}{k !}(n \sqrt{2 m})^{k} \\
& =e^{n \sqrt{2 m}} .
\end{aligned}
$$

\section{Concluding remarks}

In this paper, considering the eigenvalues of the $P I$-matrix, upper and lower bounds are established for the $P I$-Estrada index of a graph as a function of various combinatorial and spectral invariants of the given graph. Considering this matrix, a future problem is to obtain a generalization for directed graphs.

\section{Acknowledgments}

We would like to thank the anonymous referees for careful reading of our manuscript and for invaluable comments. J. Rodríguez was supported by MINEDUC-UA project, code ANT-1899 and Funded by the Initiation Program in Research Universidad de Antofagasta, INI-19-06.

\section{References}

[1] A. Alhevaz, M. Baghipur, Y. Shang, On generalized distance Gaussian Estrada index of graphs, Symmetry 11 (2019) Art\# 1276.

[2] A. Alhevaz, M. Baghipur, Y. Shang, Merging the spectral theories of distance Estrada and distance signless Laplacian Estrada indices of graphs, Mathematics 7 (2019) Art\# 995.

[3] M. J. N. Arani, Sharp bounds on the PI and vertex PI energy of graphs, MATCH Commun. Math. Chem. 65 (2011) $123-130$. 
[4] M. J. N. Arani, G. H. F. Tabar, M. Mirzagar, Sharp bounds on the PI spectral radius, Iranian J. Math. Chem. 1 (2010) $111-117$.

[5] H. Bamdad, New lower bounds for Estrada index, Bull. Malays. Math. Sci. Soc. 39 (2016) 683-688.

[6] S. B. Bozkurt, D. Bozkurt, Randić energy and Randić Estrada index of a graph, European J. Pure Appl. Math. 5 (2012) 88-99.

[7] R. Carbó-Dorca, Smooth function topological structure descriptors based on graph-spectra, J. Math. Chem. 44 (2008) 373-378.

[8] K. C. Das, I. Gutman, Bound for vertex PI index in terms of simple graph parameters, Filomat 27 (2013) 1583-1587.

[9] H. Deng, On the PI index of a graph, MATCH Commun. Math. Comput. Chem. 60 (2008) 649-657.

[10] S. S. Dragomir, A survey on Cauchy-Bunyakovsky-Schwarz type discrete inequalities, J. Inequal. Pure Appl. Math. 4 (2003) 1-142.

[11] E. Estrada, Characterization of the folding degree of proteins, Bioinformatics 18 (2002) 697-704.

[12] E. Estrada, Characterization of the amino acid contribution to the folding degree of proteins, Proteins 54 (2004) $727-737$.

[13] E. Estrada, N. Hatano, Statistical-mechanical approach to subgraph centrality in complex networks, Chem. Phys. Lett. 439 (2007) $247-251$.

[14] E. Estrada, J. A. Rodríguez-Velázquez, Subgraph centrality in complex networks, Phys. Rev. E 71 (2005) Art\# 056103.

[15] E. Estrada, J. A. Rodríguez-Velázquez, Spectral measures of bipartivity in complex networks, Phys. Rev. E 72 (2005) Art\# 046105.

[16] A. Jahanbani, Albertson energy and Albertson-Estrada index of graphs, J. Linear Topol. Algebra 8 (2019) 11-24.

[17] A. Jahanbani, Hermitian energy and Hermitian Estrada index of digraphs, Asian-Eur. J. Math. 13 (2020) Art\# 2050116.

[18] A. Jahanbani, H. H. Raz, On the harmonic energy and Estrada index of graphs, Math. Aspects Topol. Indices 1 (2019) 1-20.

[19] P. E. John, P. V. Khadikar, A method of computing the PI index of benzenoid hydrocarbons using orthogonal cuts, J. Math. Chem. 42 (2007) $37-45$.

[20] P. V. Khadikar, On novel structural descriptor PI, Nat. Sci. Lett. 23 (2000) 113-118.

[21] P. V. Khadikar, P. P. Kale, N. V. Deshpande, S. Karmarkar, V. K. Agrawal, Novel PI indices of hexagonal chains, J. Math. Chem. 29 (2001) 143-150.

[22] P. V. Khadikar, S. Karmarkar, V. K. Agrawal, Relationships and relative correlation potential of the Wiener, Szeged and PI indices, Nat. Acad. Sci. Lett. 23 (2000) 165-170.

[23] P. V. Khadikar, S. Karmarkar, V. K. Agrawal, A novel PI index and its applications to QSPR/QSAR studies, J. Chem. Inf. Comput. Sci. 41 (2001) 934-949.

[24] P. V. Khadikar, S. Karmarkar, R. G. Varma, The estimation of PI index of polyacenes, Acta Chim. Slov. 49 (2002) $755-771$.

[25] J. A. Peña, I. Gutman, J. Rada, Estimating the Estrada index, Linear Algebra Appl. 427 (2007) $70-76$.

[26] N. J. Rad, A. Jahanbani, I. Gutman, Zagreb energy and Zagreb Estrada index of graphs, MATCH Commun. Math. Comput. Chem. 79 (2018) $371-386$.

[27] N. J. Rad, A. Jahanbani, R. Hasni, Pentacyclic graphs with maximal Estrada index, Ars Combin. 133 (2017) 133-145.

[28] J. Rodríguez, A note on new bounds for the Estrada Index, Linear Algebra Appl. 580 (2019) 121-127.

[29] Y. Shang, Biased edge failure in scale-free networks based on natural connectivity, Indian J. Phys. 86 (2011) 485-488.

[30] Y. Shang, Local natural connectivity in complex networks, Chinese Phys. Lett. 28 (2011) Art\# 068903.

[31] Y. Shang, Perturbation results for the Estrada index in weighted networks, J. Phys. A 44 (2011) Art\# 075003.

[32] Y. Shang, The Estrada index of evolving graphs, Appl. Math. Comput. 250 (2015) 415-423.

[33] R. Todeschini, V. Consonni, Handbook of Molecular Descriptors, Wiley-VCH, Weinheim, 2000.

[34] R. Todeschini, V. Consonni, Molecular Descriptors for Chemoinformatics, Wiley-VCH, Weinheim, 2009. 